\title{
FASTNESS OF DYED FABRICS TO DRY CLEANING
}

\author{
By A. S. Eichlin
}

\section{ABSTRACT}

Data on the behavior of dyes in actual dry cleaning have not been available. It is the purpose of this paper to supply this information.

A number of representative dyestufis on wool, silk, cotton, rayon, and union fabrics were subjected to two tests. In the first a moisture-free solvent was used, and in the second the solvent contained 0.1 per cent free moisture and 0.01 per cent alkali. The apparatus used in making the tests was designed as a convenient substitute for a commercial dry-cleaning machine. The results are given in tabular form.

CONTENTS

I. Introduction Page

II. Purposes and scope

III. Acknowledgments

IV. Apparatus....... 40

V. Testing procedure

VI. Discussion of results

VII. Conclusion.-1... 51

\section{INTRODUCTION}

The fastness of dyestuffs to most of the agencies and operations entering into the dry-cleaning process has already been established. The behavior of dyes on fabrics toward acids, alkalies, steaming, pressing, and ironing is known and can be ascertained from the catalogues of the dye manufacturers. The action of dry-cleaning solvents and soaps on dyed fabrics has not been systematically studied.

It is probable that any type of dyestuff will at one time or another be used on textile materials which will be subjected to the dry-cleaning process. The dye may occur on the principal fabric or on the linings, threads, or decorative effects on garments or household materials which are customarily dry-cleaned. A slight change in color resulting from dry cleaning would not be particularly serious in some instances, but any tendency to bleed is always a potential danger because of the liability of staining other materials in the same load.

This study of the action of dry-cleaning solvents and soaps on dyed fabrics was undertaken by the research associate at the bureau from the National Association of Dyers \& Cleaners at the request of the association. It may well be stated here that, on account of the hundreds and even thousands of dyed materials handled every day in dry-cleaning plants, it was not considered practicable to study any 
procedure whereby the dry cleaner would attempt to group the dyed materials into their respective fastness or dyestuff classes. In some plants it is customary in the prespotting operation to test for the presence of basic dyes on certain kinds of materials before applying spotting agents. It is believed that data obtained from this systematic study of the effects of dry cleaning on dyed fabrics will be useful not only to the dry cleaner, but also to the dyestuff manufacturer and to the fabric dyer.

\section{PURPOSES AND SCOPE}

The purpose of this study was to test the fastness of dyed fabrics $(a)$ to dry-cleaning solvents and soaps, and $(b)$ to dry-cleaning solvents and soaps to which small amounts of water and alkali were added.

Many dyestuffs are identical in composition but are sold under different trade names by the various companies. To test all dyes manufactured would thus have involved needless duplication and would have served no useful purpose. Accordingly, American manufacturers were requested to submit sample dyeings of the types of dyes in which they specialized, together with dyeings of the dyestuffs which they made exclusively. In a few instances, as will be noted in the tables, duplications occurred; but these served as check tests. It is to be noted that only American dyestuffs are included; an attempt to obtain dyeings from a large importer of foreign-made dyestuffs was not successful.

\section{ACKNOWLEDGMENTS}

An acknowledgment of appreciation for whole-hearted interest and cooperation is extended to the dye manufacturers ${ }^{1}$ who provided the sample dyeings. Thanks are also due C. W. Schoffstall, formerly chief of the textile section, for his helpful suggestions.

\section{APPARATUS}

The apparatus used in making these tests is pictured in Figure 1. The machine was designed as a convenient substitute for a commercial dry-cleaning machine. It has a capacity of six 1-liter bottles. The rotor is mounted on a shaft so geared to a motor that the rotor has a speed of 30 revolutions per minute. The bottles are corked and are held in position by a spring clamping lock. The bottles rest securely in holes countersunk in the base of the rotor.

\footnotetext{
1 American Anilinc Products Co.; Geigy Co. (Inc.); John Campbell \& Co.; Celanese Corporation of America; E. I. Du Pont De Nemours \& Co.; National Aniline \& Chemical Co.; Newport Chemical Works (Inc.); Zinsser \& Co.
} 
B. S Journal of Research, RP80

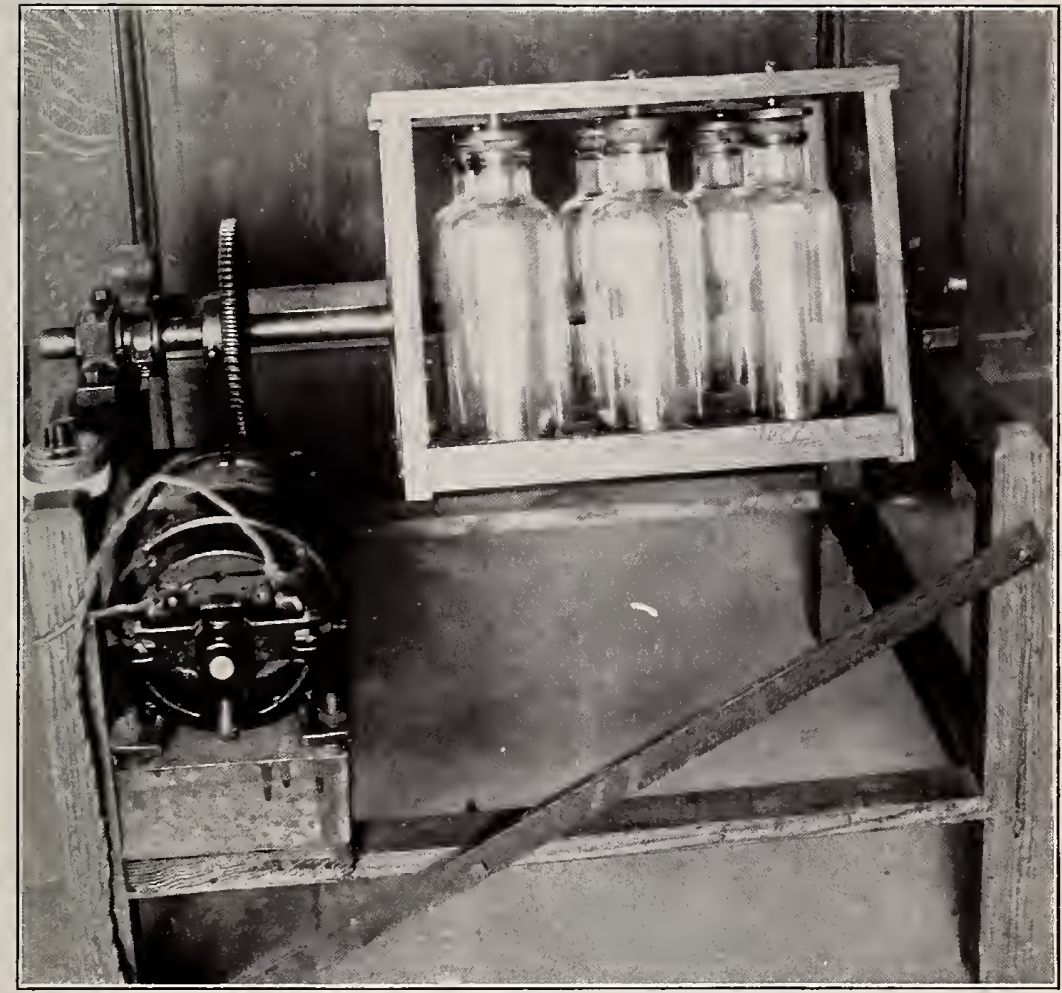

Figure 1.-Machine for laboratory dry-cleaning tests

40 


\section{TESTING PROCEDURE}

Two series of tests were made. In the first the solvent was moisture free. The soap solutions prepared from this solvent contained only the small amount of moisture naturally present in the soaps. In the second series the solvent contained 0.1 per cent water and 0.01 per cent sodium hydroxide. The soaps were prepared with the solvent containing these amounts of water and alkali. Although this content of water and alkali may appear excessive, analyses of several samples of solvents received from various cleaners showed that the use of solvents containing the above percentages is not unusual in practice.

A sample of dyed fabric 10 square inches in area was placed in each bottle, together with small portions (about $1 / 4$ square inch) of cotton, wool, rayon, and weighted and unweighted silk to be used in judging staining. In order to simulate to a close degree the mechanical action encountered in a dry-cleaning machine, four small pieces of toweling were placed in each bottle. Enough dry-cleaning solvent to cover the cloth, about $50 \mathrm{ml}$, was then added and the bottles placed in the machine. The solvent employed in this investigation was in strict conformity with specifications for Stoddard solvent. ${ }^{2}$ The machine was run for 15 minutes. The bottles were then removed, the solvent poured off, and a 0.5 per cent solution of benzene soap having a potassium oleate base added. The machine was again run for a 15-minute period, at the expiration of which the bottles were removed and the soap solution poured off. A 0.5 per cent solution of alcoholic benzene soap was next added and the machine run for an additional 15 minutes. The bottles were removed and this solution poured off. The samples were finally given a rinse in solvent for 10 minutes, extracted, and dried for 45 minutes at $120^{\circ} \mathrm{F}$.

The test procedure was repeated with fresh portions of the dyeings, using the solvent and soaps containing water and alkali. After the completion of each test, the portions of undyed fabrics were examined to determine whether or not they had become stained. The treated dyed sample was compared with the original untreated sample in order to detect possible color changes. Any coloration of the clear solvent or the soap solutions was noted at the end of each operation.

In Table 1 are listed dyes which showed no change either in moisture and alkali-free solvent or in solvent containing 0.1 per cent water and 0.01 per cent caustic soda. The dyes are divided into eight classes, namely, direct, acid, basic, mordant, sulphur, vat, developed, and ice. The dyes in each class behave similarly toward the same fiber, and the method of their application is, in general, the same. Descriptions

\footnotetext{
2Stoddard Solvent (Dry Cleaning), Commercial Standard CS3-28. Obtainable from the Superintendent of Documents, Gorernment Printing Office, Washington, D. C., for 10 cents.
} 
of these classes and methods of application can be obtained from any standard book on dyeing.

In column 1 are listed the serial numbers assigned for reference purposes in this study.

Column 2 contains the Colour Index numbers of the dyes obtained from The Colour Index published by the Society of Dyers \& Colourists (England, 1924).

This index should be consulted for data on chemical structure, methods of application, properties, uses, patent references, and trade names. The Year Book of the American Association of Textile Chemists \& Colorists lists the American manufacturers of these dyes by Colour Index number. The annual census of dyes of the United States Tariff Commission gives similar data.

Dyes which have been assigned no numbers in the Colour Index are listed by name only. The manufacturers of these dyes can be found by consulting the Year Book of the American Association of Textile Chemists \& Colorists.

Column 3 contains the names of the dyes which were tested.

In column 4 are listed the various fibers which were dyed with the dyes tabulated in column 3. The letters "C," "W," "S," "WtdS," and " $R$ " denote cotton, wool, silk, weighted silk, and rayon, respectively.

TABLE 1.-Dyes which showcd no color change in either moisture-free and alkalifree solvent or in solvent containing 0.01 per cent caustic soda and 0.1 per cent watcr

DIRECT COLORS

\begin{tabular}{r|r|l|l|l}
\hline $\begin{array}{r}\text { Serial } \\
\text { No. }\end{array}$ & $\begin{array}{c}\text { Colour } \\
\text { Index } \\
\text { No. }\end{array}$ & & Name of dye tested & Fiber on wbich \\
dyed
\end{tabular}


TABLE 1.-Dyes which showed no color change in either moisture-free and alkalifree solvent or in solvent containing 0.01 per cent caustic soda and 0.1 per cent water-Continued

DIRECT COLORS-Continued

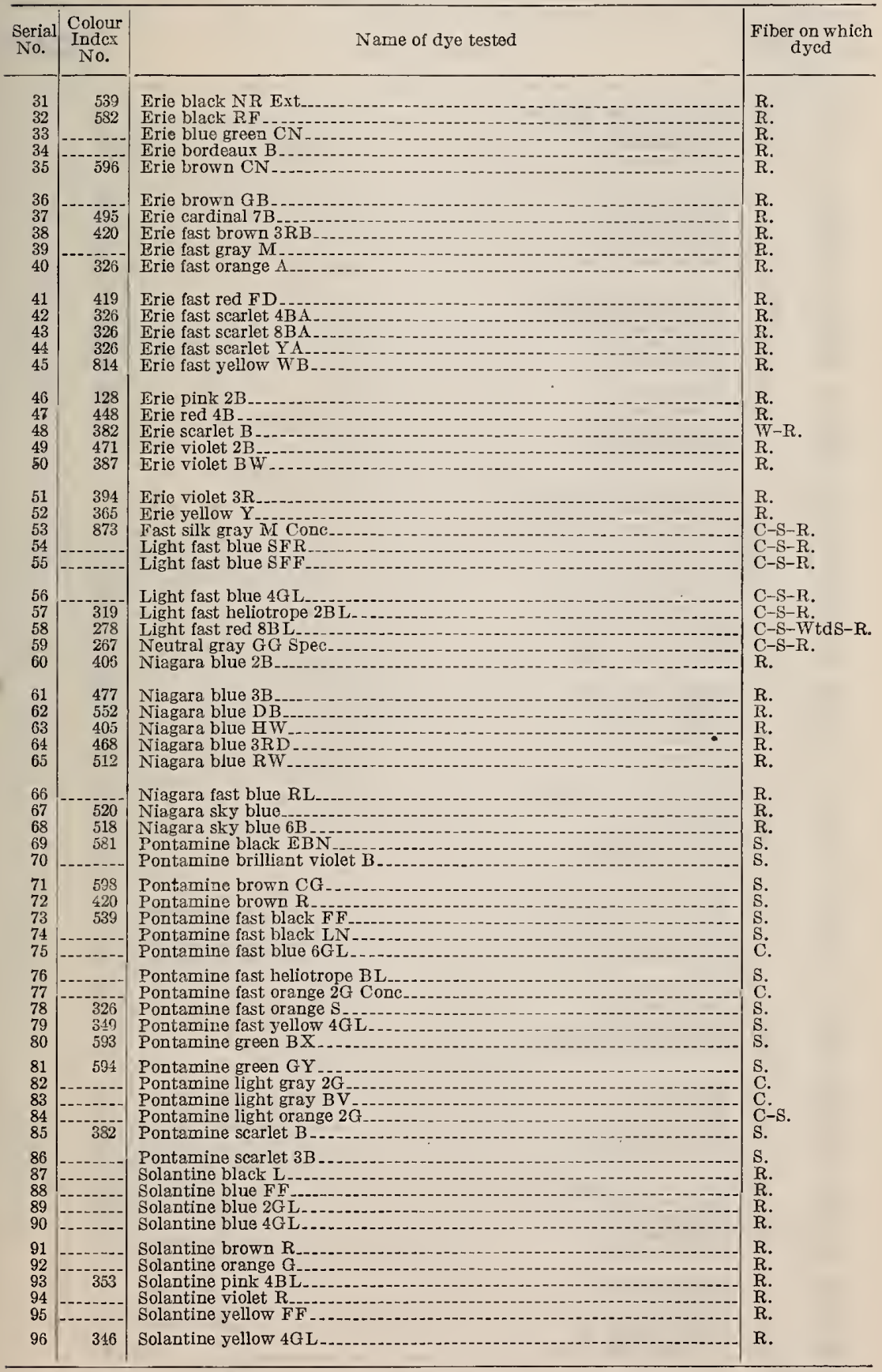

$\mathrm{C}=$ cotton, $\quad \mathrm{S}=$ silk. $\quad$ WtdS=weighted silk. $\quad \mathrm{R}=$ rayon. $\quad \mathrm{W}=$ wool. 
TABLE 1.-Dyes which showed no color change in either moisture-free and alkalifree solvent or in solvent containing 0.01 per cent caustic soda and 0.1 per cent water-Continued

ACID COLORS

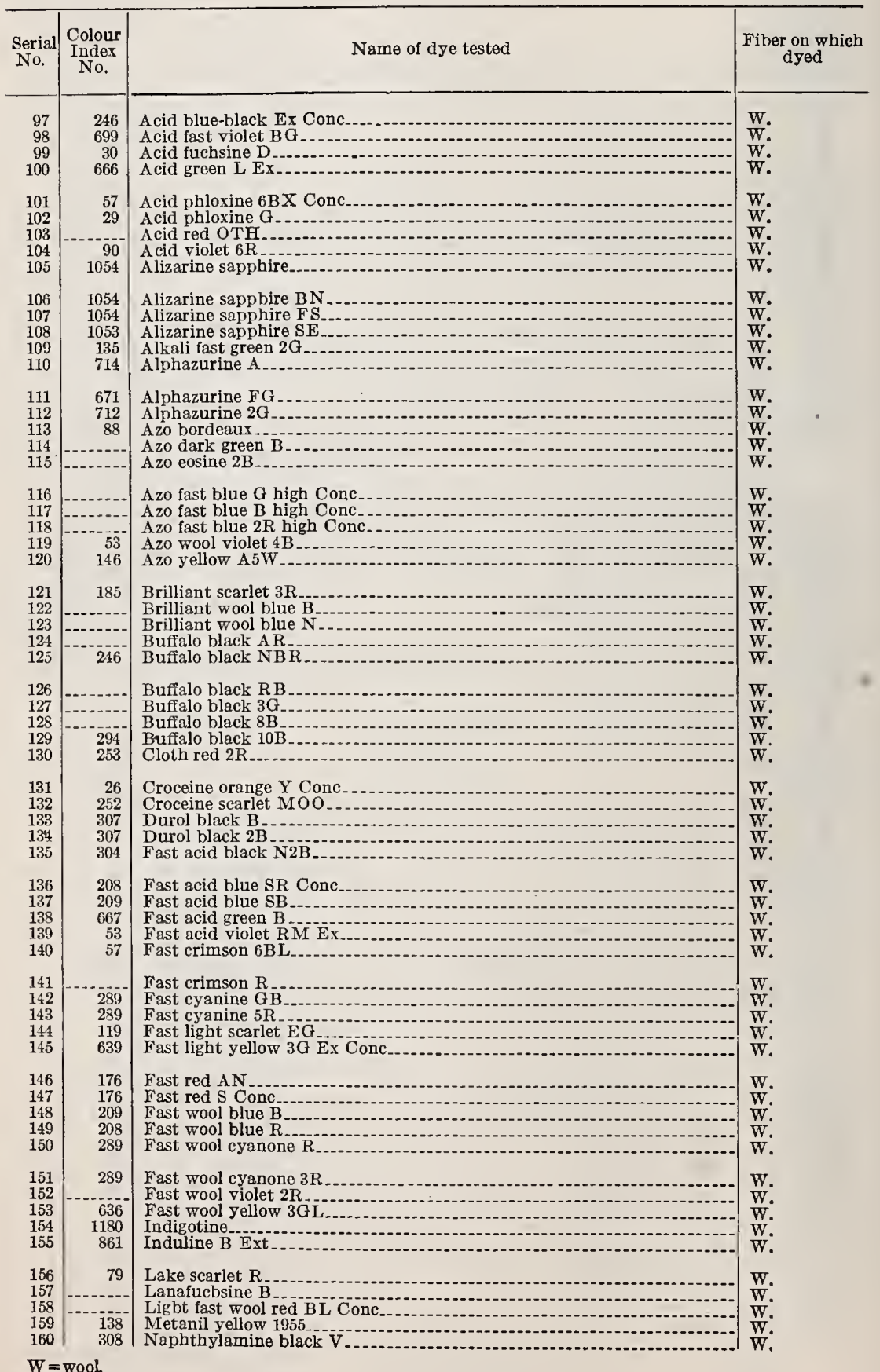


TABLE 1.-Dyes which showed no color change in either moisture-free and alkalifree solvent or in solvent containing 0.01 per cent caustic soda and 0.1 per cent water-Continued

\section{ACID COLORS-Continued}

\begin{tabular}{|c|c|c|c|}
\hline $\begin{array}{l}\text { Serial } \\
\text { No. }\end{array}$ & $\begin{array}{c}\text { Colour } \\
\text { Index } \\
\text { No. }\end{array}$ & Name of dye tested & $\begin{array}{c}\text { Fiber on wbicb } \\
\text { dyed }\end{array}$ \\
\hline 161 & 801 & Quinoline vellow & w \\
\hline 162 & 234 & Resorcine brown $\mathrm{R}$ & W. \\
\hline 163 & 235 & Resorcine brown $\mathrm{RN}$ & W. \\
\hline 164 & ......... & Wool black B & W. \\
\hline 16.5 & $-\ldots$. & Wool black GRF $\ldots .$. & W. \\
\hline 166 & & Wool blue CG. & W. \\
\hline 167 & & Wool green B... & W. \\
\hline 168 & 737 & $u^{\prime}$ & W. \\
\hline 169 & 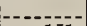 & Wool navy $B$ & W. \\
\hline 170 & 151 & Wool orange A. Conc & W. \\
\hline 171 & 27 & Wool orange $2 G$ cryst & W. \\
\hline 172 & 184 & Wool red $40 \mathrm{~F}$ & W. \\
\hline 173 & 280 & Wool scarlet BR & W. \\
\hline 174 &.--- & Wool violet $B$ & W. \\
\hline 175 & 698 & Wool violet $4 \mathrm{BN}$ & W. \\
\hline 176 & 640 & Wool yellow Ex Conc...... & W. \\
\hline
\end{tabular}

\section{MORDANT AND ACID-MORDANT COLORS}

\begin{tabular}{|c|c|c|c|}
\hline 177 & & Acid alizarine blue B. & W. \\
\hline 178 & & Alizarine black pow der & W. \\
\hline 179 & 1085 & Alizarine blue-black $3 B$ & W. \\
\hline 180 & 1035 & Alizarine brown OR & W. \\
\hline 181 & & Alizarine brown $5 \mathrm{R}$ Ext. & W. \\
\hline 182 & 1078 & Alizarine cyanine green CG Ext & W. \\
\hline 183 & 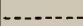 & Antbracene chrome brown 2BL & W. \\
\hline 184 & $--10=-$ & Antbracene cbrome brown RL & W. \\
\hline 185 & 1085 & Anthraquinone blue black B & W. \\
\hline 186 & 180 & Azo cbrome blue B (chromed dyed) & W. \\
\hline 187 & 180 & A zo cbrome blue $B$ (dyed acid) & W. \\
\hline 188 & 179 & Azo rubine $\mathrm{R}$ (cbrome dyed) & W. \\
\hline 189 & 179 & Azo rubine $\mathrm{R}$ (dyed acid) & W. \\
\hline 190 & 179 & Azo rubine Ex (dyed acid) & W. \\
\hline 191 & & Cbromate brown $\mathrm{R}$. & W. \\
\hline 192 & 299 & Chrome black F... & W. \\
\hline $19 \overline{3}$ & 40 & ( & W. \\
\hline 194 & 431 & Chrome red $\mathrm{A} 4 \mathrm{~B}$ & W. \\
\hline 195 & 262 & Clotb red B $\mathrm{B}$ & W. \\
\hline 196 & & Clotb red $\mathrm{R}$ & W. \\
\hline 197 & & Fast chrome brown EB. & W. \\
\hline 198 & 56 & Fast fuchsine $6 \mathbf{B}$ & W. \\
\hline 199 & 29 & Fast fucbsine $\mathrm{G}$ & W. \\
\hline 200 & & Fast fucbsine GR & W. \\
\hline 201 & & Hastings fast milling scarlet & W. \\
\hline
\end{tabular}

\section{SULPHUR COLORS}

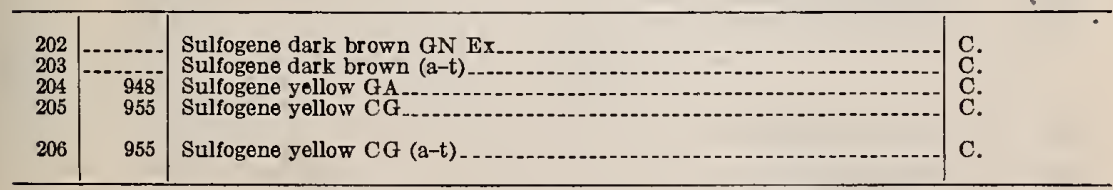

$$
\mathrm{W}=\mathrm{w} \text { ool. }
$$

$\mathrm{C}=\operatorname{cotton}$ 
TABLE 1.-Dyes which showed no color change in either moisture-free and alkalifree solvent or in solvent containing 0.01 per cent caustic soda and 0.1 per cent water-Continued

VAT COLORS

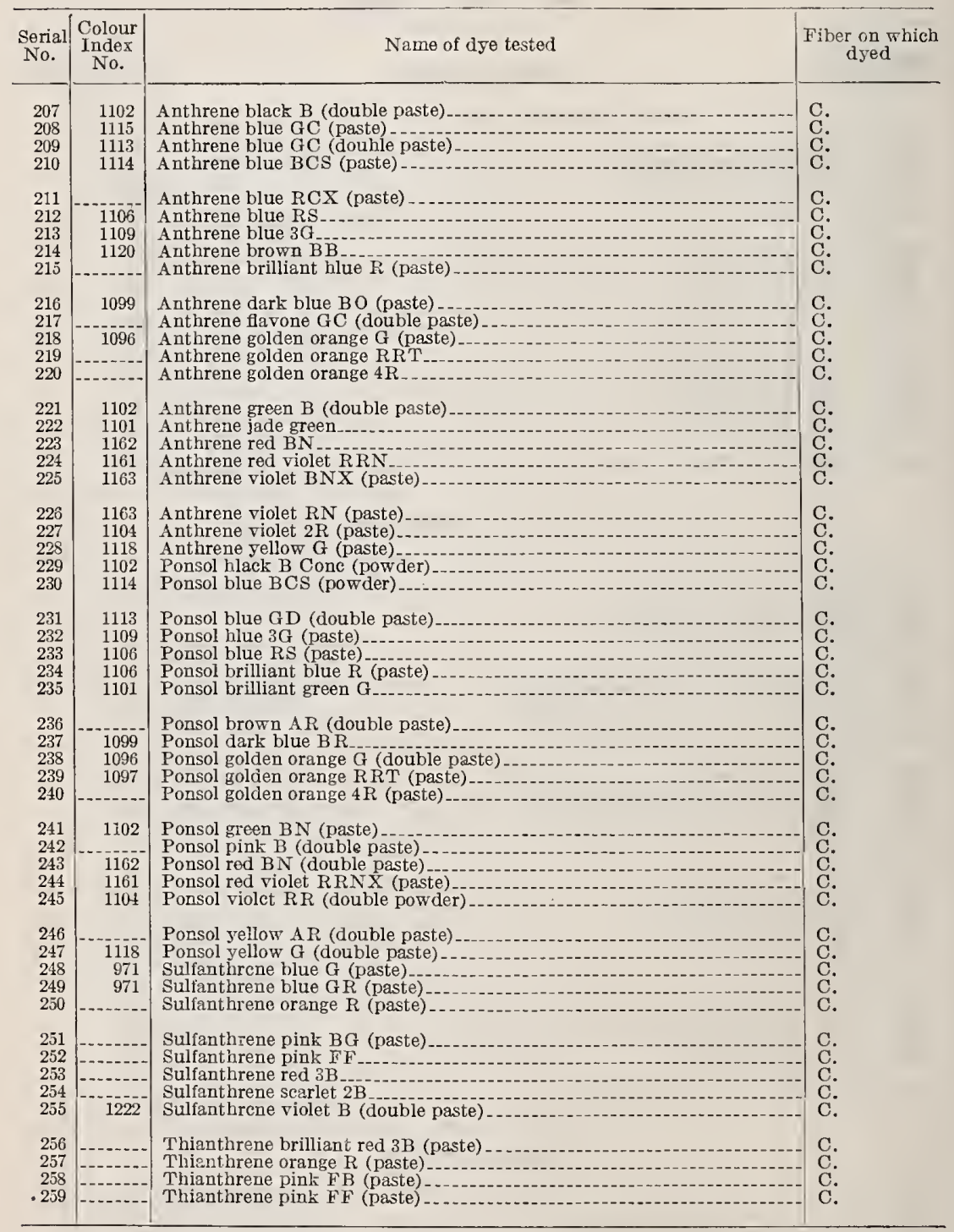

\section{DEVELOPED COLORS}

\begin{tabular}{|c|c|c|c|}
\hline 260 & 401 & Diazo black BHSW & C. \\
\hline 261 & 401 & Diazo black BHSW (DevTN) & \\
\hline 262 & 317 & Diazo blue BR & $\mathrm{C}-\mathrm{S}$. \\
\hline 263 & & Diazo blue $3 \mathrm{G}$ & $\mathrm{C}-\mathrm{S}$. \\
\hline 264 & 317 & Diazo blue $2 \mathrm{RL}$ & $\mathrm{C}-\mathrm{S}$. \\
\hline 265 & 812 & Primuline Ex Conc. & $\mathrm{C}-\mathrm{R}$. \\
\hline 266 & $324 \mathrm{~A}$ & Diazo scarlet $A$ & C. \\
\hline 267 & $324 \mathrm{~A}$ & Diazo scarlet $\mathrm{R}$ & C. \\
\hline
\end{tabular}

$\mathrm{C}=$ cotton.

$\mathrm{S}=\mathrm{silk}$.
$\mathrm{R}=$ rayon. 
TABLE 1.-Dyes which showed no color change in either moisture-free and alkalifree solvent or in solvent containing 0.01 per cent caustic soda and 0.1 per cent water-Continued

ICE COLORS

\begin{tabular}{|c|c|c|c|}
\hline $\begin{array}{l}\text { Serial } \\
\text { No. }\end{array}$ & $\begin{array}{l}\text { Colour } \\
\text { Inder } \\
\text { No. }\end{array}$ & Name of dye tested & $\begin{array}{c}\text { Fiber on which } \\
\text { dyed }\end{array}$ \\
\hline $\begin{array}{l}268 \\
269\end{array}$ & $\begin{array}{l}69 \\
69\end{array}$ & $\begin{array}{l}\text { Naphthanil AS red G base- } \\
\text { Naphthanil BS red G base }-\end{array}$ & C. \\
\hline
\end{tabular}

$\mathrm{C}=$ Cotton.

In Table 2 are recorded those dyes which showed a slight color change only.

Columns 1, 2, and 3 are the same as in Table 1.

Columns 4 and 5 show the results obtained from the tests. A dash under a fiber heading denotes that that particular fiber was not dyed and consequently could not be tested.

TABLE 2.-Dyes which showed only a slight color change

DIRECT COLORS

\begin{tabular}{|c|c|c|c|c|c|c|c|c|c|c|}
\hline \multirow[t]{2}{*}{$\begin{array}{c}\text { Serial } \\
\text { No. }\end{array}$} & \multirow{2}{*}{$\begin{array}{c}\text { Col- } \\
\text { our } \\
\text { Index } \\
\text { No. }\end{array}$} & \multirow[t]{2}{*}{ Name of dye tested } & \multicolumn{4}{|c|}{$\begin{array}{l}\text { Color ehange when } \\
\text { treated with mois- } \\
\text { ture-free and alkali- } \\
\text { free solvent }\end{array}$} & \multicolumn{4}{|c|}{$\begin{array}{l}\text { Color change when } \\
\text { treated with solvent } \\
\text { containing } 0.01 \text { per } \\
\text { cent caustic soda and } \\
0.1 \text { per cent water }\end{array}$} \\
\hline & & & $\mathrm{C}$ & $\mathrm{S}$ & WtdS & $\mathbf{R}$ & $\mathrm{C}$ & $S$ & WtdS & $\mathbf{R}$ \\
\hline 270 & 415 & Direct orange $\mathrm{R}$ & $\mathrm{N}$ & - & $\mathrm{N}$ & $\mathrm{N}$ & $\mathrm{N}$ & - & $\mathbf{N}$ & $\mathrm{S}$ \\
\hline 271 & 682 & Direct yellow G & $\mathrm{N}$ & $\mathrm{N}$ & 一 & $\bar{T}$ & $\mathrm{~N}$ & 一 & $\mathrm{S}$ & $\bar{a}$ \\
\hline 272 & & Erie catechine $3 \mathrm{G}$ & - & - & - & $N$ & 一 & 二 & - & $\underset{S}{S}$ \\
\hline $\begin{array}{l}273 \\
274\end{array}$ & 487 & $\begin{array}{l}\text { Erie catechine G } \\
\text { Erie orange } Y\end{array}$ & 二 & 二 & 二 & $\stackrel{N}{N}$ & 二 & 二 & 二 & $\stackrel{S}{S}$ \\
\hline
\end{tabular}

SULPHUR COLORS

\begin{tabular}{|c|c|c|c|c|c|c|c|c|c|c|}
\hline 275 & & Sulfogene brilliant blue $2 \mathrm{G}$ Conc. & $\mathrm{N}$ & - & - & $\mathrm{S}$ & - & - & - & - \\
\hline 276 & & Sulfogene brilliant blue $6 \mathrm{BS}$. & $\mathbf{N}$ & - & - & $S$ & - & - & - & 一 \\
\hline 277 & 1006 & Sulfogene brilliant green $2 G_{-}$ & $\mathrm{S}$ & - & 一 & $\mathrm{S}$ & 一 & - & - & 一 \\
\hline 278 & 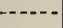 & Sulfogene direet blue BRS. & N & 二 & 二 & S & 二 & 二 & 二 & 二 \\
\hline $\begin{array}{l}279 \\
280\end{array}$ & & $\begin{array}{l}\text { Sulfogene fast green B } \\
\text { Sulfogene fast green B }(\mathrm{a}-\mathrm{t})\end{array}$ & $\underset{S}{N}$ & 二 & 二 & S & 二 & 二 & 二 & 二 \\
\hline 281 & 940 & Sulfogene golden brown G... & $\mathbf{N}$ & 一 & - & $\mathrm{S}$ & - & 一 & - & - \\
\hline 282 & 940 & Sulfogene golden brown $\mathrm{G}(\mathrm{a}-\mathrm{t})$ & $\mathrm{S}$ & - & - & $\mathrm{S}$ & - & - & - & - \\
\hline 283 & 940 & Sulfogene golden brown $\mathrm{R}$ & S & 一 & $\tau$ & S & - & - & 一 & - \\
\hline 284 & 940 & Sulfogene golden brown $R(a-t)$ - & $\mathrm{S}$ & - & - & $\mathbf{S}$ & - & - & - & 一 \\
\hline 285 & $-\cdots-\cdot-$ & Sulfogene indigo blue G Conc.-.-. & $\mathrm{N}$ & - & 一 & $\mathbf{S}$ & 一 & 一 & 一 & - \\
\hline 286 & & Sulfogene navy blue GL Conc. & $\mathbf{N}$ & - & - & S & - & - & - & - \\
\hline 287 & - & Sulfogene navy blue RL Conc. - & $\underset{\mathbf{N}}{\mathbf{N}}$ & - & - & $\underset{S}{S}$ & - & 二 & 二 & 二 \\
\hline 288 & & Sulfogene ochre $G$ & $\begin{array}{c}2 \\
\mathrm{~S}\end{array}$ & 二 & 二 & S & 二 & 二 & 二 & 二 \\
\hline 290 & & Sulfogene olive drab Y..... & $\stackrel{5}{S}$ & 二 & - & S & 二 & - & - & 二 \\
\hline 291 & 948 & Sulfogene yellow GA $(a-t)--$ & $\mathrm{N}$ & - & - & $\mathrm{S}$ & 一 & 一 & 一 & 一 \\
\hline
\end{tabular}

VAT COLORS

\begin{tabular}{l|l|l|l|l|l|l|l|}
\hline 292 & 1133 & Ponsol red AFF ..................... & $\mathrm{N}$ & - & - & $\mathrm{s}$ & - \\
\hline
\end{tabular}


TABLE 2.-Dyes which showed only a slight color change-Continued DEVELOPED COLORS

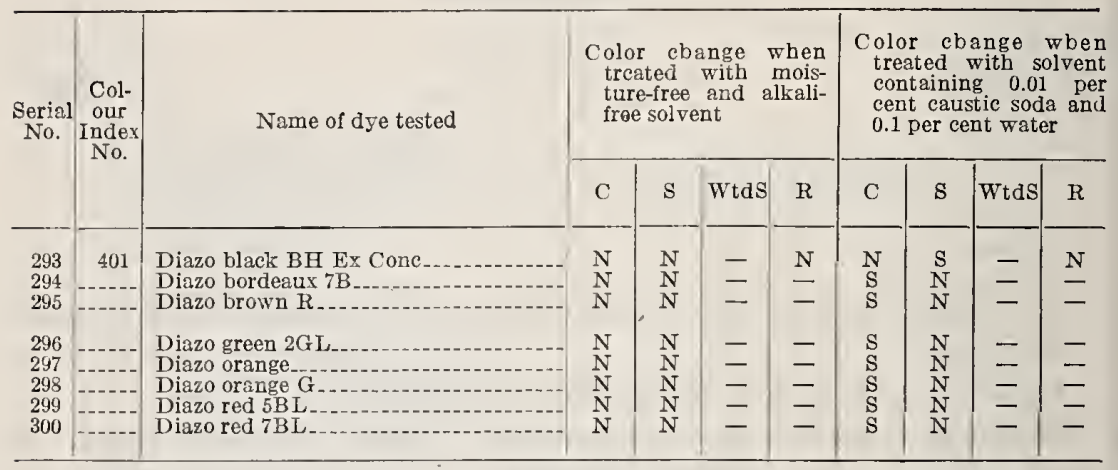

ICE COLORS

\begin{tabular}{|c|c|c|c|c|c|c|c|c|c|c|}
\hline 301 & 499 & Naphthanil AS blue B base.. & $\mathrm{N}$ & - & - & - & $\mathrm{S}$ & - & — & - \\
\hline 302 & 82 & Naphthanil AS garnet B base... & $\mathrm{N}$ & - & - & - & $\mathrm{S}$ & - & - & - \\
\hline 303 & 82 & Naphthanil BS garnet.-.-.-....... & $\mathbf{N}$ & - & - & - & $\mathrm{S}$ & - & - & - \\
\hline 304 & 38 & Napbtbanil AS orange $R$ base & IT & - & - & - & $\mathrm{S}$ & - & - & 一 \\
\hline 305 & 118 & Naphtbanil AS scarlet $\mathrm{R}$ base & $\mathrm{N}$ & - & - & - & $\mathrm{S}$ & - & - & - \\
\hline 306 & 118 & Naphtbanil BS scarlet.... & $\mathrm{N}$ & - & - & - & $\mathrm{S}$ & - & - & - \\
\hline
\end{tabular}

$\mathrm{N}=$ no color change.

$\mathrm{S}=$ slight color change.

In Table 3 are listed those dyes which showed a decided color change. The letter "D" denotes a decided color change.

TABLE 3.-Dyes which showed a decided color change

DIRECT COLORS

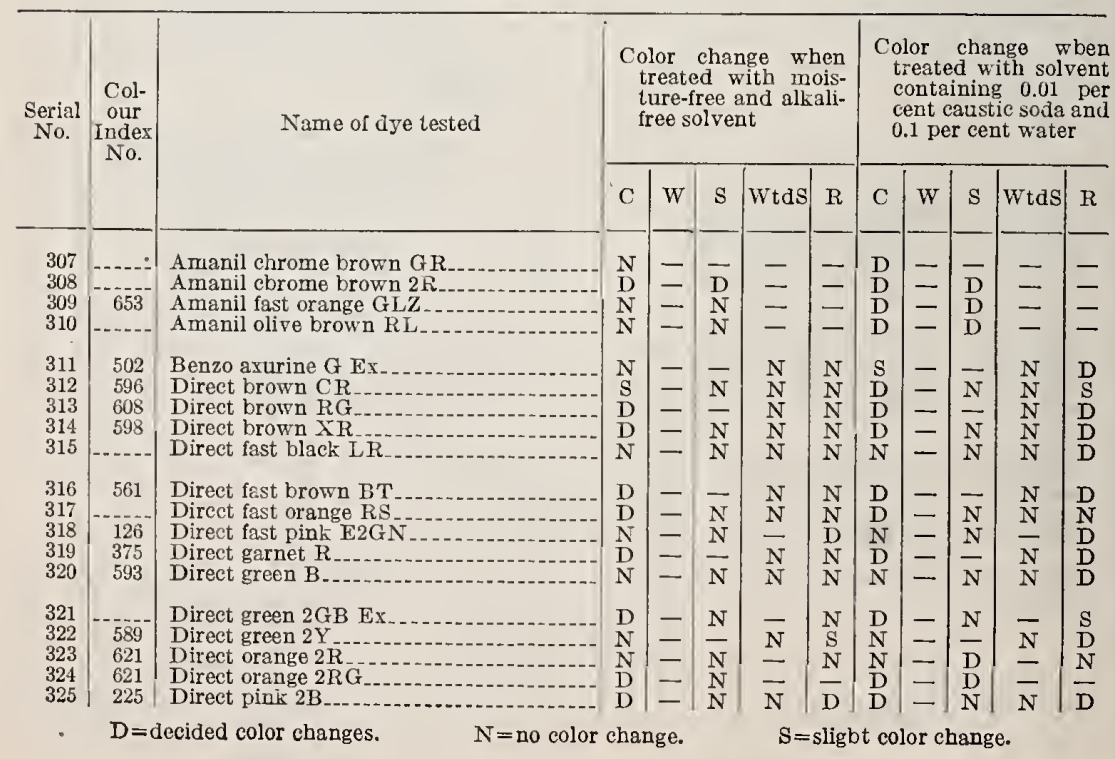


TABLE 3.-Dyes which showed a decided color change-Continued

DIRECT COLORS-Continued

\begin{tabular}{|c|c|c|c|c|c|c|c|c|c|c|c|c|}
\hline \multirow[t]{2}{*}{$\begin{array}{l}\text { Serial } \\
\text { No. }\end{array}$} & \multirow{2}{*}{$\begin{array}{c}\text { Col- } \\
\text { our } \\
\text { Index } \\
\text { No. }\end{array}$} & \multirow[t]{2}{*}{ Name of dye tested } & \multicolumn{5}{|c|}{$\begin{array}{l}\text { Color change when } \\
\text { treated with mois- } \\
\text { ture-free and alkali- } \\
\text { free solvent }\end{array}$} & \multicolumn{5}{|c|}{$\begin{array}{l}\text { Color change when } \\
\text { treated with solvent } \\
\text { containing } 0.01 \text { per } \\
\text { cent caustic soda and } \\
0.1 \text { per cent water }\end{array}$} \\
\hline & & & $\mathrm{C}$ & W & $\mathrm{S}$ & Wtds & $\mathrm{R}$ & C & W & $\mathrm{S}$ & Wtds & $\mathrm{R}$ \\
\hline 326 & 596 & Erie brown $3 \mathrm{GN}$ & - & - & - & - & $\mathrm{N}$ & - & - & - & - & \\
\hline $326 \mathrm{~A}$ & & Erie catechin & - & - & - & - & $\mathrm{N}$ & - & 二 & 二 & 二 & D \\
\hline 327 & 598 & Erie fast brown GR & - & - & - & - & $\mathrm{N}$ & - & - & - & - & $\mathrm{D}$ \\
\hline 328 & 375 & Erie garnet RB & - & 一 & - & - & $\mathbf{N}$ & - & - & - & - & $\bar{D}$ \\
\hline 329 & 593 & Erie green $M T$. & - & - & - & - & $\mathrm{N}$ & - & - & - & - & $\mathrm{D}$ \\
\hline 330 & 583 & Erie green WT & - & 一 & - & 一 & $\mathrm{N}$ & - & - & - & - & $\mathrm{D}$ \\
\hline 331 & & Light fast brown $\mathrm{R}$ & $\mathrm{D}$ & 一 & $\mathrm{N}$ & - & $\mathrm{N}$ & $\mathrm{D}$ & 一 & $\mathrm{D}$ & - & $\mathrm{D}$ \\
\hline & & Light fast brow & $\mathrm{s}$ & - & $\mathrm{N}$ & $\overline{-1}$ & $\mathrm{~N}$ & $\mathrm{D}$ & - & $\mathrm{N}$ & $\bar{\tau}$ & $\mathrm{D}$ \\
\hline 333 & 423 & Light fast yellow $4 \mathrm{GL}$ & $\mathrm{S}$ & - & - & $\mathrm{N}$ & $\mathrm{N}$ & $\mathrm{D}$ & - & - & $\mathrm{N}$ & $\mathrm{S}$ \\
\hline 334 & 502 & Niagara blue G Conc-- & - & 一 & - & - & $\mathbf{N}$ & - & - & - & - & $\mathrm{D}$ \\
\hline 335 & & Niagara blue NR & - & 一 & - & - & $\mathrm{N}$ & - & - & 一 & - & $\mathrm{D}$ \\
\hline 336 & 515 & Niagara blue $R_{-}$ & - & 一 & - & - & $\mathbf{N}$ & - & - & - & - & $\mathrm{D}$ \\
\hline $336 \mathrm{~A}$ & & Solantine red $8 \mathrm{~B} \mathrm{LN}$ & - & 一 & - & - & $\mathrm{N}$ & - & - & 一 & - & $\overline{\mathrm{D}}$ \\
\hline
\end{tabular}

BASIC COLORS

\begin{tabular}{|c|c|c|c|c|c|c|c|c|c|c|c|c|}
\hline 337 & 655 & Auramine $\mathrm{O}_{-} \ldots$ & $\mathrm{N}$ & - & - & - & - & $\mathrm{D}$ & - & - & - & - \\
\hline 338 & 332 & Basic brown $\mathrm{B} \mathrm{R}$ & $\mathrm{D}$ & $\longrightarrow$ & - & - & 一 & $\mathrm{D}$ & - & 一 & - & - \\
\hline 339 & 21 & Chrysoidine $\mathrm{R}$ & $\mathrm{D}$ & - & - & - & - & $\mathrm{D}$ & - & - & - & - \\
\hline 340 & 681 & Crystal violet & $\mathrm{N}$ & - & $\mathrm{N}$ & 一 & 一 & $\mathrm{D}$ & $\longrightarrow$ & $\mathrm{D}$ & $\leftarrow$ & - \\
\hline 341 & 680 & Methyl violet NE. & $\mathrm{D}$ & - & - & - & - & $\mathrm{D}$ & 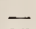 & - & - & - \\
\hline 342 & 778 & Phloxine B & - & $\mathbf{N}$ & $\mathbf{N}$ & - & 一 & - & $N$ & $\mathrm{D}$ & - & - \\
\hline 343 & 749 & Rhodamine $\mathbf{B} \mathbf{E x} \ldots \ldots$ & $\mathrm{D}$ & $\mathbf{N}$ & $\mathrm{N}$ & $\mathbf{N}$ & 一 & $\mathrm{D}$ & $\mathrm{N}$ & $\mathrm{D}$ & $\mathrm{N}$ & 一 \\
\hline 344 & 779 & Rose bengal & $\overline{-1}$ & $\mathrm{~N}$ & $\mathrm{~N}$ & - & 一 & - & $\mathrm{D}$ & $\mathrm{D}$ & - & 一 \\
\hline 345 & 841 & Safranine $\mathrm{T} \mathrm{Ex}$ & $\mathbf{N}$ & 一 & $\mathrm{N}$ & 一 & 一 & $\mathrm{D}$ & - & $\mathrm{D}$ & 一 & 一 \\
\hline 346 & 815 & Thioflavine, T G Ex. & - & $\mathbf{N}$ & $\mathrm{N}$ & $\mathbf{N}$ & $\mathrm{D}$ & - & $\mathbf{N}$ & $\mathrm{D}$ & $N$ & $\mathrm{D}$ \\
\hline 347 & 729 & Victorial blue $\mathrm{BX}$ & $\mathrm{D}$ & $\mathrm{N}$ & $\mathrm{N}$ & - & - & $\mathrm{D}$ & $\mathrm{S}$ & $\mathrm{D}$ & - & - \\
\hline 348 & 657 & Victorial green & $\mathrm{N}$ & 一 & $\mathrm{D}$ & 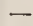 & - & $\mathrm{D}$ & - & $\mathrm{D}$ & - & - \\
\hline
\end{tabular}

SULPHUR COLORS

\begin{tabular}{|c|c|c|c|c|c|c|c|c|c|c|c|c|}
\hline $\begin{array}{l}349 \\
350\end{array}$ & $\begin{array}{l}1012 \\
1012\end{array}$ & $\begin{array}{l}\text { Sulfogene bordeaux BR } \\
\text { Sulfogene bordeaux BR }(a-t)\end{array}$ & $\begin{array}{l}\mathrm{D} \\
\mathrm{D}\end{array}$ & - & - & - & - & $\begin{array}{l}\mathrm{D} \\
\mathrm{D}\end{array}$ & - & - & - & - \\
\hline 351 & 1012 & Sulfogene bordeaux $5 \mathrm{~B}$ & D & 一 & $\longrightarrow$ & 一 & - & $\mathrm{D}$ & 一 & - & 一 & - \\
\hline & 1012 & fogene bordeaux $5 \bar{B}(a-t)$ & $\mathrm{D}$ & 一 & - & - & 一 & D & $\longrightarrow$ & - & - & 一 \\
\hline 53 & & Sulfogene brilliant green $4 \mathrm{GX}$ & S & 一 & 一 & 一 & - & $\mathrm{D}$ & - & - & 一 & - \\
\hline 3 & 988 & Sulfogene carbon 2B Conc. & N & - & - & 一 & - & $\mathrm{D}$ & 一 & - & 一 & - \\
\hline 355 & 978 & Sulfogene carbon $8 \mathrm{G}$ Conc. & $\mathrm{N}$ & 一 & - & 一 & 一 & $\mathrm{D}$ & - & - & 一 & 一 \\
\hline 356 & & rbon HXX.... & $\mathbf{N}$ & 一 & - & 一 & - & $\mathrm{D}$ & - & 一 & 一 & 一 \\
\hline 57 & 978 & rbon $\mathrm{M}$ Conc & $\mathbf{S}$ & 一 & 一 & 一 & 一 & $\mathrm{D}$ & 一 & - & - & -- \\
\hline & 978 & supra_._._-_. & $\mathbf{S}$ & $\hookleftarrow$ & 一 & 一 & - & $\mathrm{D}$ & 一 & - & - & 一 \\
\hline & 978 & 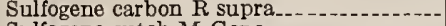 & $\mathbf{S}$ & 一 & - & 一 & 一 & $\mathrm{D}$ & 一 & 一 & 一 & 一 \\
\hline 360 & $-\cdots$ & Sulfogene cutch M Conc. & $\mathrm{S}$ & 一 & 一 & 一 & - & $\mathrm{D}$ & 一 & 一 & 一 & - \\
\hline 36 & & $(a-t)$ & $\mathbf{S}$ & - & - & - & - & $\mathrm{D}$ & 一 & - & 一 & 一 \\
\hline & & 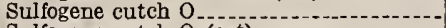 & $\mathbf{S}$ & 一 & 一 & 一 & - & $\mathrm{D}$ & - & - & 一 & 一 \\
\hline 363 & & $\mathrm{O}(\mathrm{a}-\mathrm{t})$ & $\overline{\mathbf{S}}$ & - & 一 & 一 & 一 & $\overline{\mathrm{D}}$ & 一 & 一 & 一 & 一 \\
\hline 364 & 1006 & $2 \mathrm{~B}$ & $\mathbf{N}$ & - & - & - & - & D & - & 一 & 一 & 一 \\
\hline 365 & 1006 & Sulfogene green 2B (a-t) & $\mathrm{N}$ & - & 一 & - & 一 & $\mathrm{D}$ & 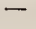 & - & 一 & 一 \\
\hline 366 & 1006 & 1 Conc_ & $\mathbf{N}$ & 一 & 一 & 一 & 一 & D & 一 & 一 & - & - \\
\hline 367 & & $\mathrm{e}$ & $\mathbf{N}$ & 一 & 一 & 一 & - & D & - & - & - & - \\
\hline 368 & & ene olive $G(a-t)$ & $\mathbf{N}$ & 一 & - & 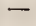 & 一 & $\mathrm{D}$ & 一 & 一 & 一 & 一 \\
\hline 369 & & ogene olive $\mathrm{GN}$ & $\mathrm{N}$ & - & - & - & - & D & 一 & - & 一 & 一 \\
\hline 370 & & Sulfogene olive GN (a-t) & $\mathbf{N}$ & 一 & - & 一 & 一 & $\mathrm{D}$ & 一 & 一 & - & 一 \\
\hline 371 & & Sulfogene olive drab Y $(a-t)$ & $\mathbf{S}$ & - & 一 & - & 一 & $\mathrm{D}$ & 一 & 一 & 一 & 一 \\
\hline
\end{tabular}


TABLE 3.-Dyes which showed a decided color change-Continued

DEVELOPED COLORS

\begin{tabular}{|c|c|c|c|c|c|c|c|c|c|c|c|c|}
\hline \multirow[t]{2}{*}{$\begin{array}{l}\text { Serial } \\
\text { No. }\end{array}$} & \multirow{2}{*}{$\begin{array}{c}\text { Col- } \\
\text { our } \\
\text { Index } \\
\text { No. }\end{array}$} & \multirow[t]{2}{*}{ Name of dye tested } & \multicolumn{5}{|c|}{$\begin{array}{l}\text { Color change when } \\
\text { treated with mois- } \\
\text { ture-free and alkali- } \\
\text { free solvent }\end{array}$} & \multicolumn{5}{|c|}{$\begin{array}{l}\text { Color change when } \\
\text { treated with solrent } \\
\text { containing } 0.01 \text { per } \\
\text { cent caustic soda and } \\
0.01 \text { per cent water }\end{array}$} \\
\hline & & & $\mathrm{C}$ & $\mathrm{w}$ & $\mathrm{s}$ & Wtds & $\mathrm{R}$ & $\mathrm{C}$ & $\mathrm{w}$ & $\mathrm{s}$ & Wtds & $\mathbf{R}$ \\
\hline 372 & 552 & Diazo black RS. & $N$ & - & 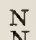 & 二 & $\mathrm{N}$ & $\stackrel{D}{N}$ & 二 & $\stackrel{N}{N}$ & 二 & $\underline{\mathrm{D}}$ \\
\hline $\begin{array}{l}373 \\
374\end{array}$ & & $\begin{array}{l}\text { Diazo brown } 20 .- \\
\text { Fast black } V_{-}\end{array}$ & N & 二 & $\mathrm{N}$ & 二 & $\vec{N}$ & $\mathrm{~s}$ & 二 & $\mathrm{S}$ & 二 & $\overline{\mathrm{D}}$ \\
\hline 375 & & Diazo orange $2 \mathrm{R}$ & $\mathbf{N}$ & - & $\mathrm{N}$ & - & & $\mathrm{S}$ & - & $D$ & - & - \\
\hline 376 & $-\ldots .$. & Diazo scarlet $2 \mathrm{BL}$ & $\mathbf{N}$ & - & $\mathrm{N}$ & - & - & S & - & $\mathrm{D}$ & - & - \\
\hline $\begin{array}{l}377 \\
378\end{array}$ & 654 & $\begin{array}{l}\text { Diazo violet } 2 \mathrm{GL} \\
\text { Diazo yellow }\end{array}$ & N & 二 & N & 二 & 二 & $\stackrel{N}{D}$ & 二 & $\mathrm{D}$ & 二 & 二 \\
\hline
\end{tabular}

ICE COLORS

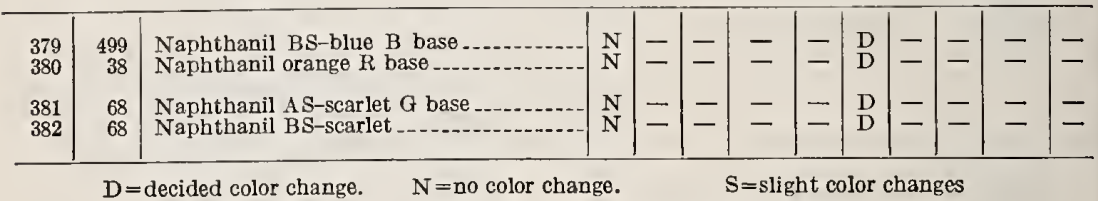

\section{DISCUSSION OF RESULTS}

The above results show that about 75 per cent of dyes of the direct class were unaffected by dry-cleaning solvents. No change was noted on dyeings made on weighted silks. Ninety per cent of the dyeings on silk, 72 per cent of the dyeings on rayon, 70 per cent of the dyeings on cotton showed no color change.

Dyeings on wool with acid, mordant, and acid-mordant colors were unaffected.

The basic colors offered poor resistance to dry cleaning. Samples Nos. 342,343 , and 346 on wool, and No. 343 on weighted silk, were unaffected. Sample No. 346, dyed on rayon and unweighted silk, was almost completely discharged. The majority of basic colors on cotton and silk stained other fabrics.

Practically all of the sulphur colors suffered an alteration in shade in both of the tests. The samples marked " $(a-t)$ " were aftertreated with copper sulphate, sodium dichromate, and acetic acid. Dyeings made with the vat colors were unchanged. Several of these colors bled in the tests but did not stain white fabrics. This was particularly true of the red, pink, and red-violet colors. The bleeding of these colors is to be expected, since the majority of the dyes of this group are somewhat soluble in the solvent.

The ice and developed colors showed no change in shade in moisturefree solvent, but in the solvent containing alkali about 65 per cent of these dyeings suffered an alteration in shade, The majority of the 
ice colors bled considerably in the tests but did not stain white materials.

In addition to the tests made above, other tests were conducted on the following dyed fabrics: Cellulose acetate rayon; weighted silk dyed with Setamine colors; mixed fabrics dyed with union colors; draperies, couch coverings, and cotton, linen, jute casement fabrics; silk velvet; and transparent velvets.

Of the 60 samples of cellulose acetate rayon tested, four samples showed a color change. One of the samples which was dyed a seasonal shade showed a slight color change in the solvent containing alkali. Two samples of printed material showed a decided color change in the solvent containing alkali. The fourth sample, a printed voile, showed a decided color change in both tests.

Weighted silk fabrics dyed with Setamine colors showed no color change in either test.

No change in shade was detected in testing 60 samples of uniondyed fabrics.

Of the 26 samples of draperies, couch coverings, and cotton, linen, and jute casement fabrics, three showed a decided color change in both tests. Two of these were cotton cretonnes and the other a cotton and rayon casement cloth.

A sample of silk velvet dyed with a basic color showed a decided color change in both tests. The color bled and tinted white silk, cotton, and rayon.

Six samples of transparent velvet showed no color change in either test.

Dyeings of acid colors on wool, sulphur colors on cotton, developed and vat colors, and direct colors on rayon were made in two strengthsthe one a light shade, the other a dark shade. This was done in order to ascertain whether or not light dyeings behaved differently than deep shades. No difference in results was noted between dyeings made in two strengths.

\section{CONCLUSION}

From the results obtained it is apparent that, although basic dyes offer poor resistance to dry cleaning, the majority of dyes which are most likely to be encountered in dry cleaning are unaffected by it. In order to minimize the danger of any change in color during dry cleaning, it is advisable to keep the solvent as free as practicable from moisture and alkali.

Washington, February 19, 1929. 